\title{
APLICATIVO DE DETERMINAÇÃO DOS PERFIS DE VELOCIDADE E TEMPERATURA NO RESFRIAMENTO ACELERADO DE CHAPAS GROSSAS
}

Antônio Adel dos Santos ' Cristóvão Nery Giacomin ${ }^{2}$

\section{Resumo}

O objetivo do trabalho foi desenvolver e aplicar modelos matemáticos para determinação dos perfis de velocidade e temperatura de chapas processadas por resfriamento acelerado, na Linha de Chapas Grossas da Usiminas em Ipatinga. Os modelos foram desenvolvidos com base na representação matemática/numérica dos fenômenos físicos envolvidos, tendo sido validados com dados de produção de 3334 chapas. Considerando-se tais modelos, os aços processados e os parâmetros de configuração da Linha da Usiminas, foi construído um aplicativo em Visual Basic, dotado de fácil acesso ao usuário. Com isso, são gerados os perfis de temperatura na espessura e ao longo do tempo para qualquer grau de aço e dimensional do laminado, possibilitando ajustes nos modelos de controle online de processo. Além disso, o aplicativo de simulação tem sido muito útil no desenvolvimento de novos graus de aço, uma vez que as variáveis de processo podem ser relacionadas à evolução térmica, que afeta diretamente as propriedades mecânicas.

Palavras-chave: Resfriamento acelerado; Simulação matemática; Chapas grossas.

\section{SIMULATION TOOL OF VELOCITY AND TEMPERATURE PROFILES IN THE ACCELERATED COOLING PROCESS OF HEAVY PLATES}

\begin{abstract}
The aim of this paper was to develop and apply mathematical models for determining the velocity and temperature profiles of heavy plates processed by accelerated cooling at Usiminas' Plate Mill in Ipatinga. The development was based on the mathematical/numerical representation of physical phenomena occurring in the processing line. Production data from 3334 plates processed in the Plate Mill were used for validating the models. A user-friendly simulation tool was developed within the Visual Basic framework, taking into account all steel grades produced, the configuration parameters of the production line and these models. With the aid of this tool the thermal profile through the plate thickness for any steel grade and dimensions can be generated, which allows the tuning of online process control models. The simulation tool has been very useful for the development of new steel grades, since the process variables can be related to the thermal profile, which affects the mechanical properties of the steels.
\end{abstract}

Keywords: Accelerated cooling; Mathematical simulation; Heavy plates.

\section{INTRODUÇÃO}

O conceito de resfriamento acelerado para otimização das propriedades mecânicas de laminados planos é relativamente antigo, sendo aplicado à laminação de tiras a quente desde o início da década de 1960. A aplicação em chapas grossas data do início da década de 1980, no Japão [I].

A partir daí, houve disseminação de sua aplicação para outros países [2-6], especialmente para o ocidente, sendo atualmente uma tecnologia utilizada na maioria das linhas de chapas grossas do mundo.
Comparativamente ao processo de laminação controlada seguida de resfriamento ao ar, o processo de resfriamento acelerado permite que aços avançados com alta resistência mecânica sem deterioração da tenacidade sejam produzidos. Para algumas aplicações específicas, tais como transporte e armazenamento de óleo e gás em ambientes com alto teor de enxofre, este processo é indispensável à obtenção de chapas de aço. Em outras aplicações, de menor restrição, graus equivalentes de aços podem ser produzidos com menor adição de elementos de liga na aciaria, reduzindo o custo.

\footnotetext{
'Engenheiro Metalurgista, D.Sc., CQE/ASQ, Centro de Tecnologia Usiminas, Ipatinga, MG, Brasil. E-mail: antonio.adel@usiminas.com ${ }^{2}$ Analista de TI, Centro de Tecnologia Usiminas, Ipatinga, MG, Brasil.E-mail: cristovao.giacomin@usiminas.com
} 
Vários aspectos precisam ser controlados durante o resfriamento acelerado em chapas grossas. É fundamental a obtenção de resfriamento relativamente homogêneo ao longo do comprimento do laminado, com simetria na espessura e largura, atendendo a uma determinada taxa de resfriamento. Nos processos industriais, as medições de temperatura são feitas sobre a superfície do laminado antes e após este processo. Os modelos de controle do processo e as eventuais intervenções manuais dos operadores são feitas considerando-se estas temperaturas, que não indicam sua variação ao longo da espessura.

A aplicação de modelos de simulação matemática offline é uma maneira muito útil de melhorar o ajuste dos modelos de controle e permitir maior entendimento do efeito das variáveis de processo nos valores obtidos de temperatura e taxas de resfriamento.

Existem numerosos trabalhos na literatura relativos ao processo de resfriamento acelerado de chapas grossas, apontando suas vantagens, aplicações e características gerais [I-8]. Muitas vezes são até mostradas curvas de resfriamento, mas de forma esquemática [7-9], sem detalhamento dos cálculos dos perfis de velocidade e de temperatura da chapa

Assim, o propósito deste trabalho é apresentar uma visão geral de um modelo matemático de cálculo dos perfis de velocidade e temperatura de chapas grossas processadas por resfriamento acelerado. $O$ modelo foi aplicado para a instalação da Usiminas, denominada CLC pelo fornecedor, a NSSMC (Nippon Steel \& Sumitomo Metal Corporation), trazendo excelentes resultados. Em parte, o sucesso de aplicação do modelo se deve à forma de sua implementação, como um aplicativo amigável e de fácil interação com o usuário, seguindo a linha de outros aplicativos já desenvolvidos na Usiminas [10-12].

\section{LINHA DE LAMINAÇÃO COM RESFRIAMENTO ACELERADO}

Na Figura I está apresentado um esquema de uma linha de laminação de chapas grossas, exemplificada para a Usiminas em Ipatinga. Equipamentos básicos são os fornos de reaquecimento, a estação de descarepação primária, HSB, os laminadores, a desempenadeira a quente e o equipamento de resfriamento acelerado. A etapa de laminação, que pode ser realizada em um ou dois laminadores, é feita na Usiminas em um laminador acabador. A concepção de desempeno a quente da chapa varia com a tecnologia aplicada [13], podendo haver uma pré-desempenadeira leve antes do resfriamento e uma pesada após. Na Usiminas, a concepção é uma desempenadeira pesada antes do CLC. Também com relação ao equipamento de resfriamento acelerado, existem concepções variando com a tecnologia desenvolvida [14]. No CLC de Ipatinga, o equipamento possui seis zonas com controle independente de vazão de água no ambiente superior e seis no inferior. Ajustes de velocidade do laminado e de relação entre as vazões de água das zonas superiores e inferiores são realizados visando obtenção do resfriamento desejado. Visando melhorar a homogeneidade de temperatura ao longo do comprimento e largura do laminado, existem controles de máscara de topo, de base e de bordas, que interceptam a incidência de água no local. Na maioria dos materiais, o resfriamento é obtido com a passagem do laminado somente uma vez pelo equipamento. Para espessuras de laminados mais elevadas, pode ser necessária oscilação no seu interior.

\section{MODELOS MATEMÁtICOS}

\section{I Perfil de Velocidade da Chapa}

O modelo do processo de resfriamento acelerado precisa considerar o fenômeno transiente de transferência de calor, em diferentes regimes, numa chapa em movimento. Então, é necessário entender e modelar o deslocamento da chapa, através dos diagramas TPV, tempo-posição-velocidade. Para isso, foram mapeadas as distâncias ao longo da linha, entre os equipamentos, sensores de posição e de temperatura, onde ocorrem mudanças de velocidade e/ou de regime de resfriamento. As regras de variação de velocidade do laminado aplicadas ao processo foram traduzidas em equações matemáticas que, por sua vez, aplicadas em algoritmos, permitem relacionar a posição e a velocidade do laminado ao longo do tempo, considerando-se topo, meio e base.

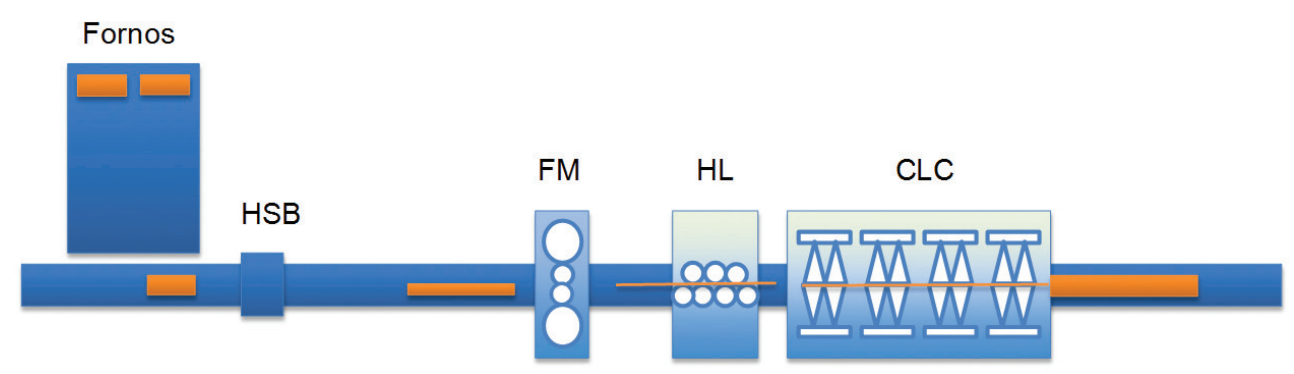

Figura I. Esquema da linha de chapas grossas da Usina de Ipatinga. HSB: descarepador primário; FM: laminador acabador; HL: desempenadeira a quente. 
Na Figura 2 são mostrados perfis esquemáticos da velocidade do laminado, considerando o topo, meio e base. As linhas verticais tracejadas na parte superior da figura denotam as distâncias mapeadas. O meio e a base do laminado estão defasados do topo, em relação à posição na linha de processo, pela metade do seu comprimento, e pelo comprimento, respectivamente. $O$ cálculo é iniciado antes da última passagem pelo $\mathrm{FM}$, no ponto de parada sob o pirômetro de entrada. Neste momento, no tempo zero, a velocidade é nula. A partir deste momento a chapa é acelerada uniformemente até a velocidade VFM de transporte para a $\mathrm{HL}$, a qual permanece até que o topo atinja determinado sensor de posição. A partir daí, ocorre desaceleração até a velocidade inicial da HL. Até o próximo sensor de posição esta velocidade é utilizada, quando ocorre aceleração até o valor VHL na entrada da HL. A partir deste ponto a velocidade é calculada pelo modelo de resfriamento do CLC. As equações de cálculo da velocidade são aplicadas ao topo do laminado e consistem de MU, movimento uniforme, e MUV, movimento uniformemente variado.

Da entrada do topo no CLC até que a base do laminado atinja o sensor de saída do CLC, ocorre aceleração não constante até a velocidade atingir o valor máximo. $A$ velocidade máxima é mantida até que a base do laminado atinja o sensor de posição final. Então, a velocidade é zerada de acordo com desaceleração constante, e retomada a velocidade de saída do CLC.

As rotinas de velocidade construídas estão acopladas com um cálculo preliminar de temperatura no resfriamento, de forma que a aceleração é calculada visando à obtenção da temperatura final de resfriamento desejada em todo laminado.
Existem sensores de medição das velocidades de entrada e de saída do CLC. Foram levantados dados de processo de 3334 chapas laminadas, incluindo-se tais velocidades, que permitiram traçar os gráficos de valores calculados com os medidos, Figura 3. O modelo de cálculo de velocidade permitiu, sistematicamente, boa previsão das relações TPV do laminado, o que foi atestado pelo erro médio de cálculo das velocidades, próximo de $3 \mathrm{~m} /$ $\mathrm{min}$, no intervalo operacional de $15 \mathrm{~m} / \mathrm{min}$ a $150 \mathrm{~m} / \mathrm{min}$.

\subsection{Modelo de Temperatura}

O modelo de temperatura é unidimensional na espessura do laminado, no meio da largura e aplicado ao topo, meio do comprimento e base do laminado. A equação que governa a transferência de calor por condução em regime transiente no interior do laminado, equação de Fourier, unidimensional na espessura, é dada em (I).

$$
\rho c \frac{\partial T}{\partial t}=\frac{\partial}{\partial x}\left(\lambda \frac{\partial T}{\partial x}\right)
$$

$\rho$ : densidade do aço $\left(\mathrm{kg} \cdot \mathrm{m}^{-3}\right)$;

c: calor específico do aço $\left(\mathrm{J} \cdot \mathrm{kg}^{-1} \cdot{ }^{\circ} \mathrm{C}^{-1}\right)$;

$\mathrm{T}$ : temperatura $\left({ }^{\circ} \mathrm{C}\right)$;

t: tempo (s);

$\lambda$ : condutividade térmica do aço $\left(\mathrm{W} \cdot \mathrm{m}^{-1} .{ }^{\circ} \mathrm{C}^{-1}\right)$;

$\mathrm{x}$ : posição na espessura $(\mathrm{m})$.

As propriedades c, $\rho$ e $\lambda$ são obtidas das tabelas BISRA [15], instituto de pesquisa que determinou tais propriedades em 22 aços. No modelo existe uma rotina que enquadra o aço a ser simulado naquele código BISRA cuja composição química mais se aproxima do aço

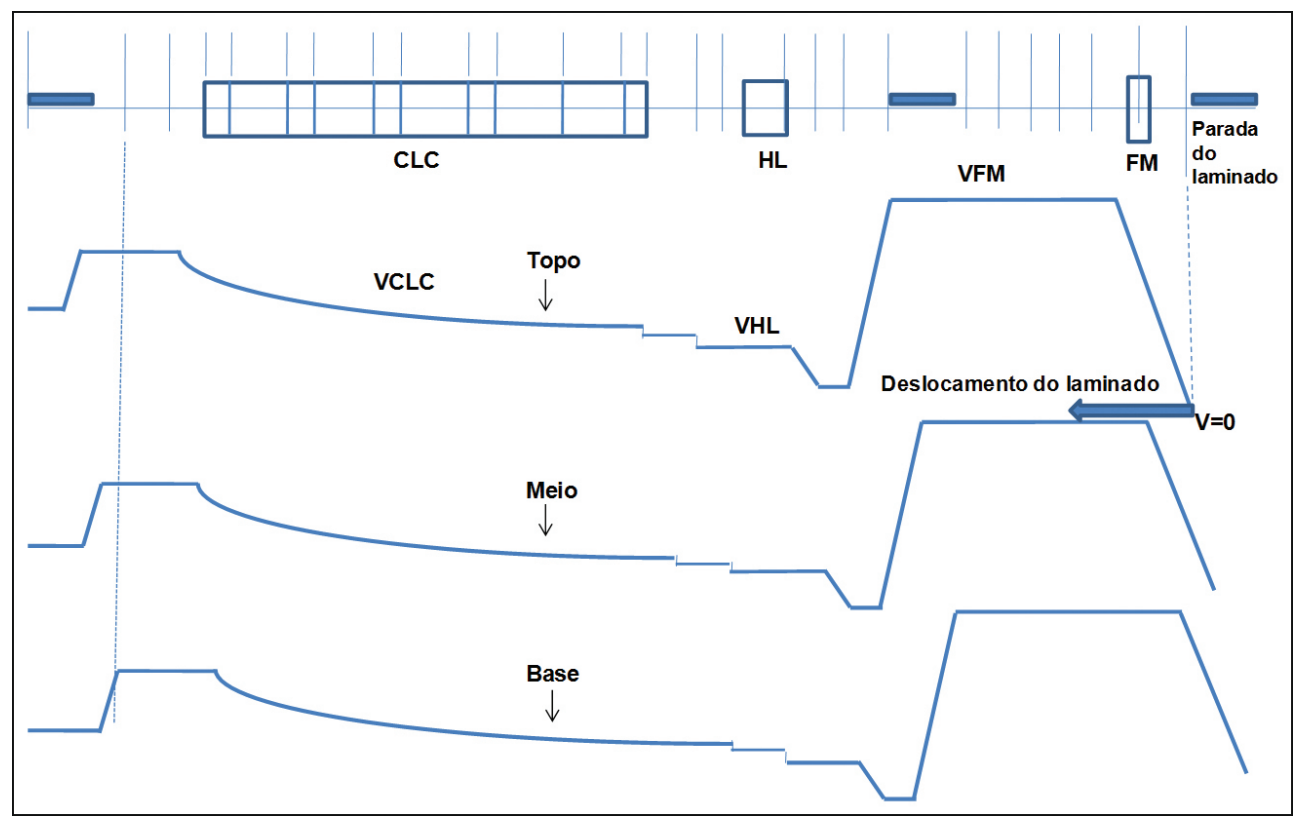

Figura 2. Perfil esquemático de velocidade do topo, meio e base do laminado desde a entrada do laminador até o final da linha CLC. 

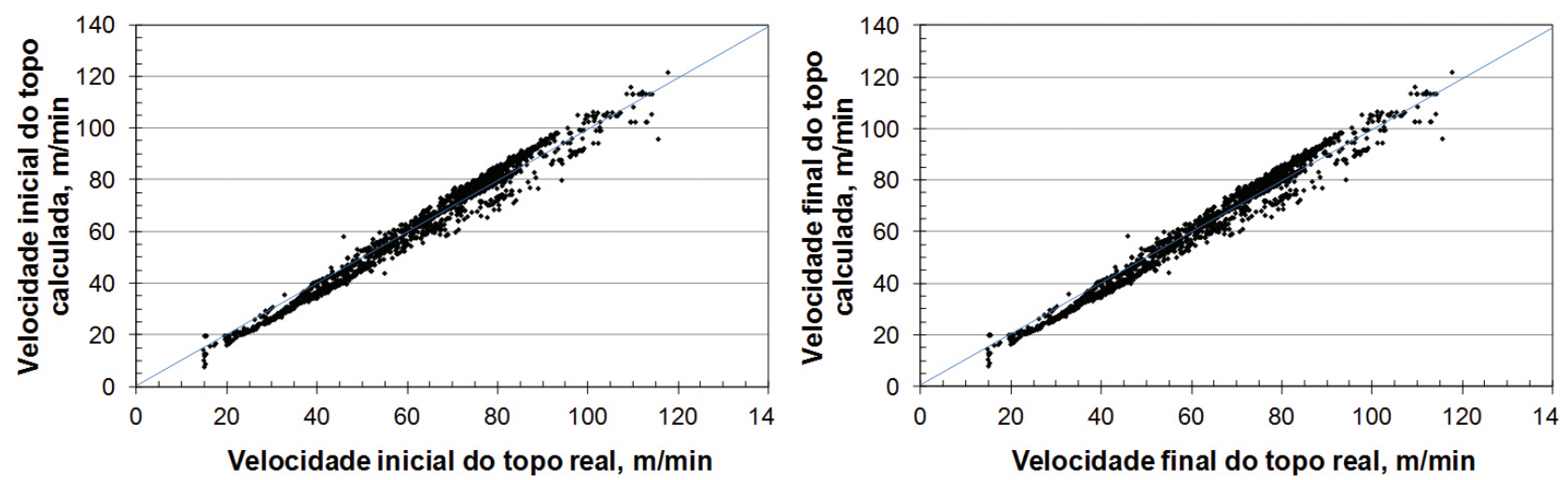

Figura 3. Gráficos comparativos de velocidade do topo do laminado, calculada e medida, na entrada e na saída do CLC.

estudado. Como os valores são tabelados em função da temperatura, a cada instante de cálculo são feitas interpolações, para a temperatura existente, entre os valores tabelados.

A condição inicial de temperatura do laminado é a temperatura do último passe de acabamento no FM, caso a opção de cálculo seja completa, ou a temperatura de início de resfriamento do CLC desejada, caso a simulação contemple apenas o CLC.

A transferência de calor da superfície do laminado para o meio ambiente, representada pelos coeficientes de transferência de calor, é a questão mais importante e complicada em modelos térmicos. No presente modelo, foram utilizadas expressões matemáticas obtidas pelo fornecedor do equipamento que consideram diferenciadamente as superfícies superior e inferior do laminado. Durante o resfriamento ao ar, o coeficiente global de transferência de calor é uma função polinomial de terceiro grau da temperatura superficial do laminado. Durante o resfriamento com água, o coeficiente de transferência de calor é determinado por um conjunto de equações considerando a temperatura superficial do laminado, a vazão de água por área de incidência, a temperatura da água e fatores relativos à classe do aço laminado.

Como tanto as propriedades termofísicas quanto os coeficientes de transferência de calor são dependentes da temperatura, a solução da equação para cálculo da evolução térmica é feita de forma numérica. Foi escolhido o método das diferenças finitas, formulação explícita, para rapidez de cálculo. Neste caso o intervalo de tempo em cada passo da iteração é controlado [16]. Alternativamente, foi implantada a solução pelo método de volumes finitos [I7], incondicionalmente convergente, mas a solução é mais demorada. Detalhes sobre tais métodos são facilmente encontrados na literatura [16-19], inclusive em outros trabalhos dos autores [10-12]. Na solução numérica são usados onze nós ao longo da espessura.

O modelo global de simulação do resfriamento acelerado, tendo como núcleos os cálculos dos perfis de velocidade e de temperatura, foram codificados em Visual Basic 6, com mais detalhes apresentados no item seguinte.
Após testes de consistência e validação dos cálculos matemáticos, o modelo foi executado para os 3334 laminados cujos dados foram coletados. O objetivo, desta vez, foi comparar os cálculos de temperatura com as medições de processo, que são realizadas na entrada e na saída do CLC.

$\mathrm{Na}$ Figura 4a é mostrado o gráfico comparativo da temperatura calculada com a visada do processo, corrigida pelo operador, para o final de resfriamento do laminado. Existe uma aproximação entre valores calculados e medidos, sendo o erro padrão da estimativa $16^{\circ} \mathrm{C}$. Porém, quando são comparados valores calculados com os medidos houve elevada dispersão, Figura 4b, com erro padrão da estimativa de $63^{\circ} \mathrm{C}$. Apesar de o acerto da temperatura final de resfriamento em faixas muito estreitas ser naturalmente um grande desafio, foi buscado melhorar a aproximação entre valores calculados, medidos e visados. Para isso, foi introduzido um módulo de otimização de parâmetros do modelo matemático, empregando-se o método simplex [20]. Na Figura 4c é mostrado um gráfico comparativo da temperatura calculada com a medida após ajuste de um parâmetro do coeficiente de transferência de calor por resfriamento forçado através de otimização. $O$ erro máximo tolerado na simulação dos 3334 laminados foi $\pm 5^{\circ} \mathrm{C}$. Houve convergência da otimização em todos os laminados. Com esta função, o modelo de controle de processo pode ser ajustado para grupos específicos de aços, atuando-se nos parâmetros do coeficiente de transferência de calor.

\section{APLICATIVO DE SIMULAÇÃO}

Os modelos matemáticos foram inseridos em um aplicativo de simulação, construído em plataforma visual, que contem: tabelas de cadastro de todos os parâmetros da linha de laminação inerentes à simulação, composições químicas e dimensionais de todos os aços passíveis de serem processados nas linhas, propriedades termofísicas dos aços e parâmetros de controle dos modelos matemáticos. As informações estão organizadas em um banco de 
dados. A interface de simulação, representada na Figura 5, é flexível e possui diversas alternativas de simulação, tais como domínio de simulação, método de solução matemática, grau e dimensional do aço, etc. A simulação é feita normalmente na forma direta, que permite o cálculo da evolução térmica do laminado em função das variáveis de entrada, incluindo a quantidade de água no resfriamento forçado. Uma alternativa interessante introduzida é o modelo de cálculo inverso, utilizando algoritmo de otimização simplex [20], que permite determinar a quantidade de água para que seja obtido um valor visado de taxa de resfriamento. Ao todo são dezoito interfaces de entrada.
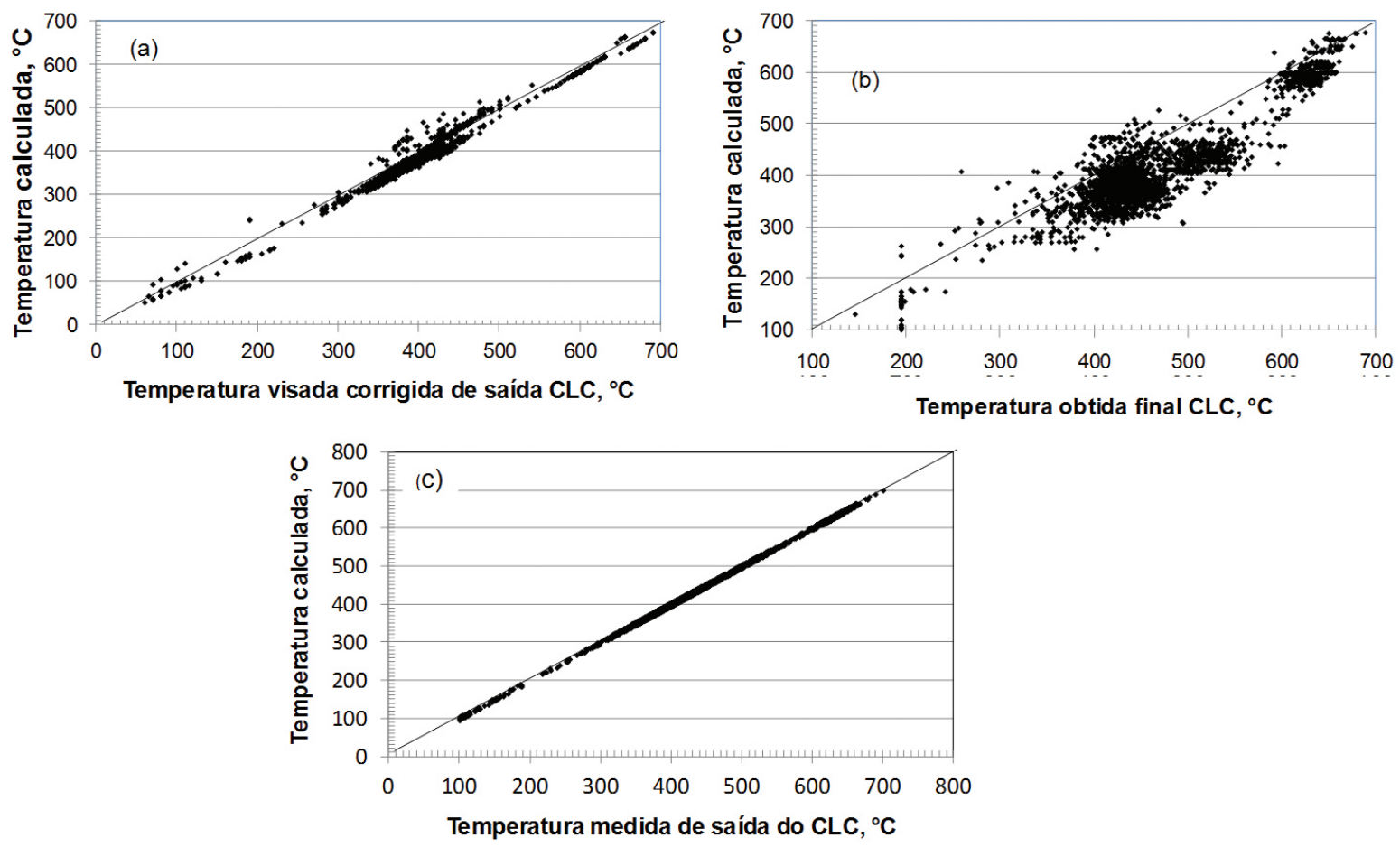

Figura 4. Gráficos comparativos de temperatura calculada e media do processo de resfriamento.

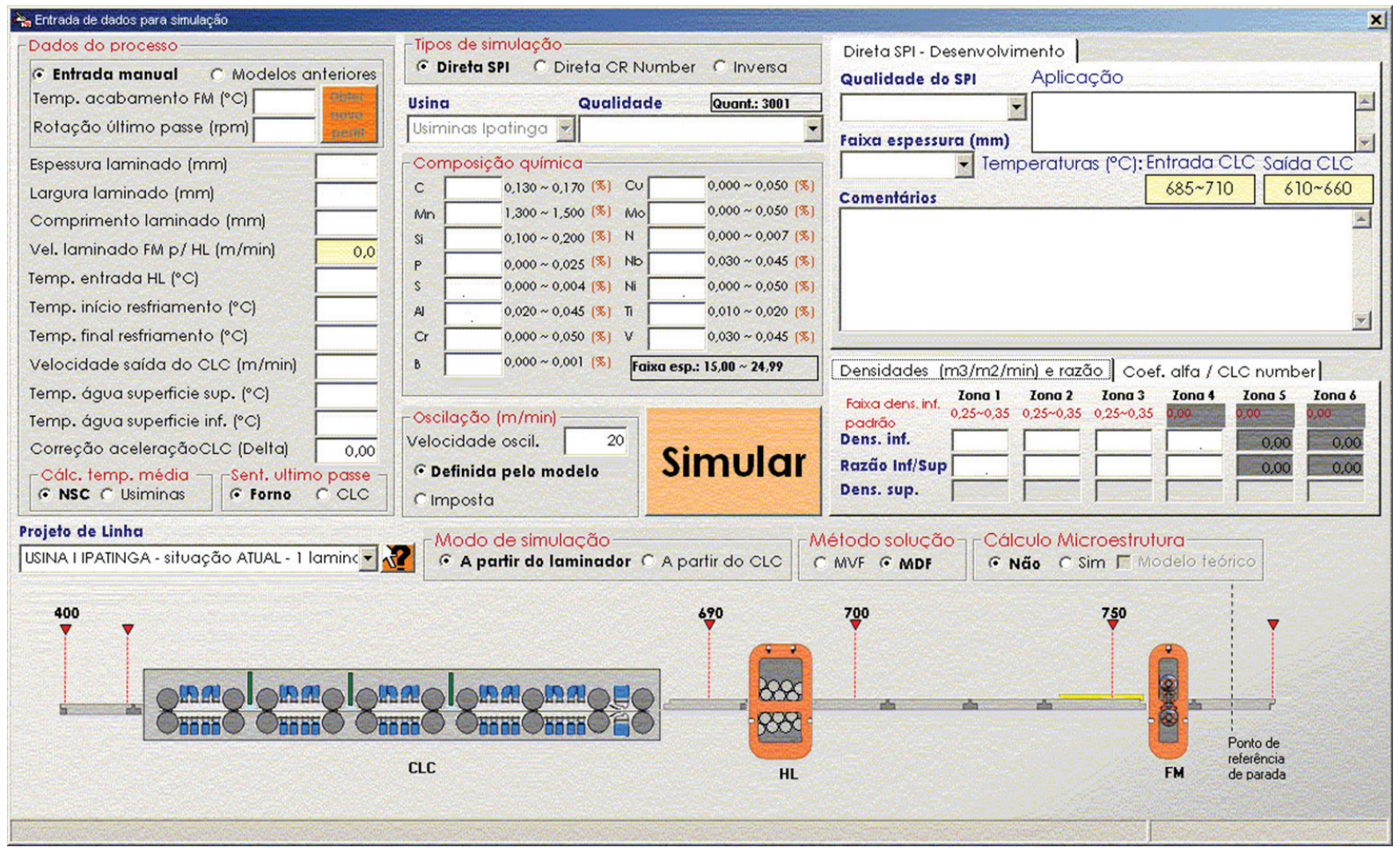

Figura 5. Interface principal de simulação do resfriamento acelerado, no aplicativo desenvolvido. 


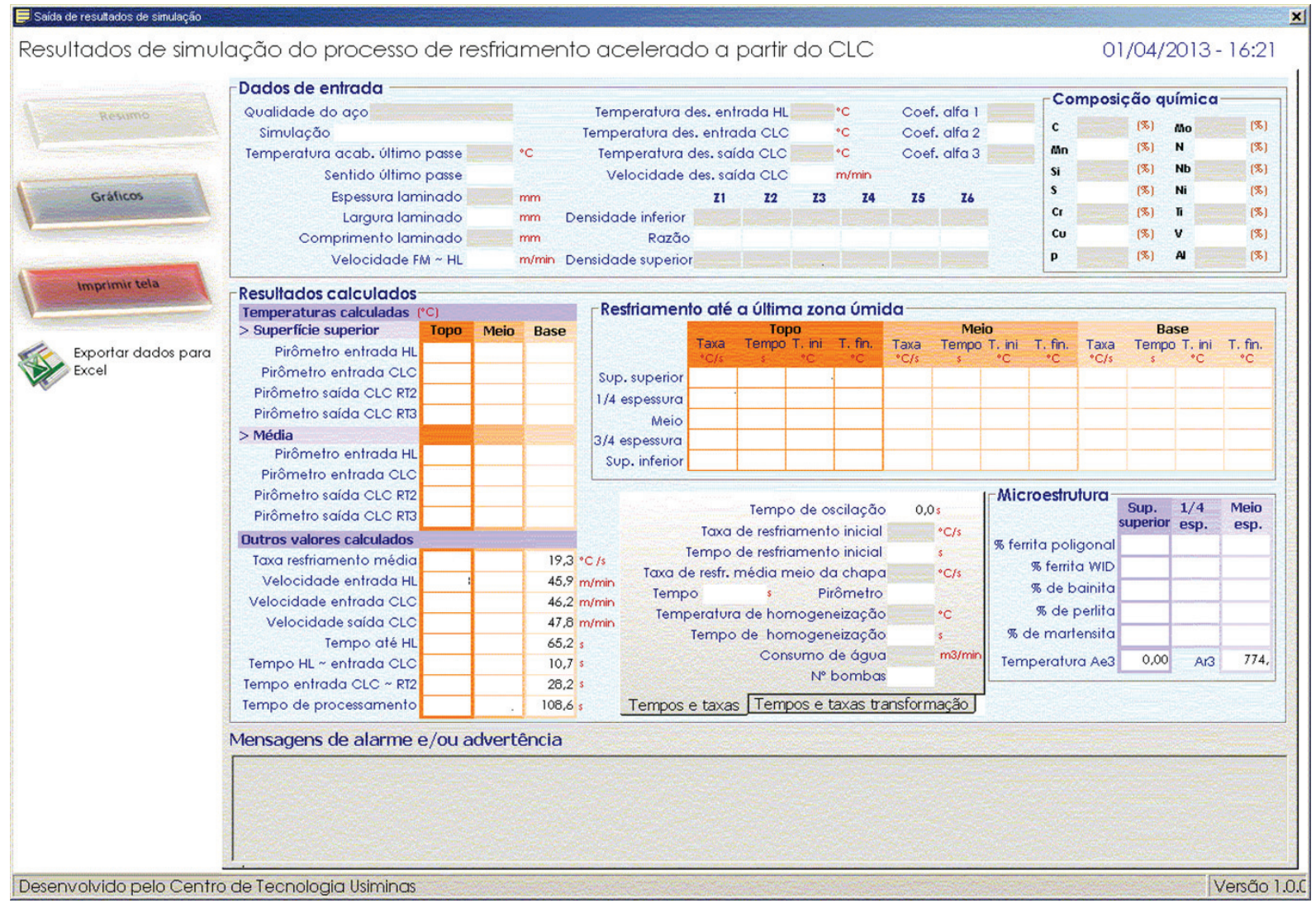

Figura 6. Interface de saída de resultados do aplicativo de simulação do resfriamento acelerado.

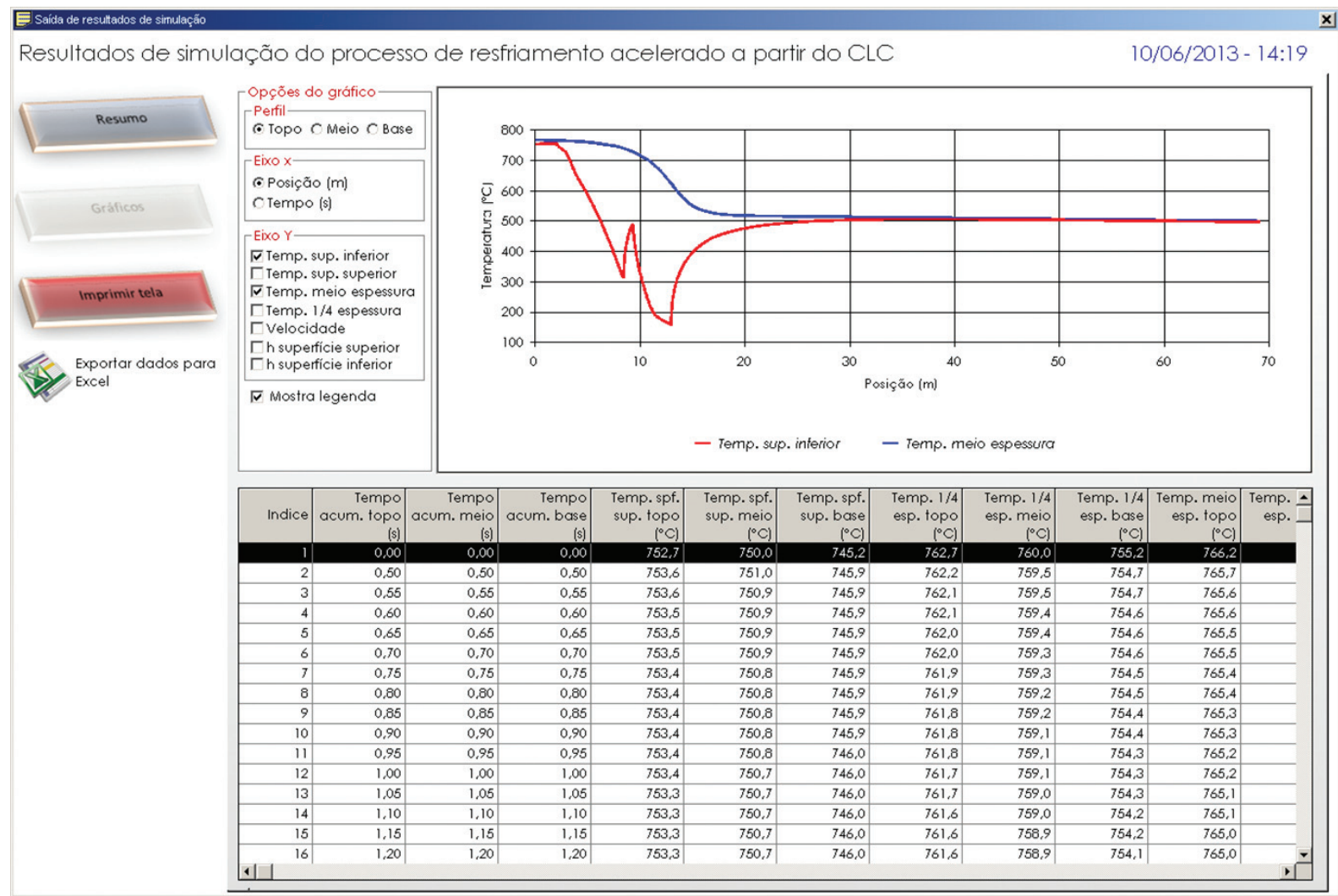

Desenvolvido pelo Centro de Tecnologia Usiminas

Versão 1.0.2

Figura 7. Gráfico exemplo dos perfis de temperatura calculados para a superfície e o meio da espessura do laminado. 
Além das simulações direta e inversa, aplicadas a cada laminado individualmente, o aplicativo pode ser usado para a simulação sequencial de um conjunto de laminados, tanto na forma direta, por exemplo, usado nas Figuras $4 \mathrm{a}$ e $4 \mathrm{~b}$, quanto na otimização de algum parâmetro do modelo visando melhorar $\circ$ acerto de temperatura de final de resfriamento, usado por exemplo na Figura 4c. $\mathrm{Na}$ interface de saída, Figura 6, são mostrados os principais dados de entrada, valores de velocidade do laminado, tempos de processo, temperaturas superficial e média em várias posições da linha e taxas de resfriamento. Os tempos e taxas de resfriamento podem ser calculados em intervalos específicos definidos pelo usuário, e são apresentados ao acionar a aba correspondente.

Os perfis de temperatura, velocidade e coeficientes de transferência de calor podem ser apresentados graficamente, como ilustra a Figura 7. Esta simulação foi apenas para a região do CLC, portanto o perfil de temperatura se restringe a este domínio. Uma série de opções é apresentada no lado esquerdo para configuração dos gráficos. Os resultados são também mostrados em tabelas que podem ser exportadas para planilhas eletrônicas, caso haja interesse do usuário em estender o seu tratamento.

\section{CONCLUSÕES}

Foram desenvolvidos e aplicados, com sucesso, modelos matemáticos de cálculo dos perfis de velocidade e temperatura do laminado durante o resfriamento acelerado na Usiminas.

Este sucesso pode ser creditado à combinação de fatores: (a) modelagem parametrizada do processo industrial em todos seus detalhes; (b) desenvolvimento de modelos matemáticos representativos dos fenômenos físicos existentes com soluções numéricas apropriadas; (c) levantamento de dados de processamento de materiais para validação dos modelos matemáticos; (d) implementação dos modelos em aplicativos privilegiando os conceitos de usabilidade no seu desenvolvimento. Com isso, foi disponibilizada para várias áreas da Empresa uma ferramenta versátil e confiável para suporte à melhoria do processo de resfriamento acelerado e ao desenvolvimento de novos aços empregando esta tecnologia.

\section{REFERÊNCIAS}

I Tsukada K, Matsumoto K, Hirabe K, Takeshige K. Application of on-line accelerated cooling (OLAC) to steel plate. In: Proceedings of the 23rd Mechanical Working and Steel Processing Conference; 198I; Pittsburgh, EUA. Englewood: AIME; 198I. p. 347-370.

2 Gräf MK, Hillenbrand HG, Peters PA. Accelerated cooling of plate for high-strength large-diameter pipe. In: Proceedings of the 4th International Steel Rolling Conference; 1987; Deauville, França. Maizières-lès Metz: IRSID ATS; 1987. p. 507-516.

3 Willmote S, Noville JF. The multi-purpose interrupted cooling process in operation in Clabecq Plate Mill. In: Proceedings of the Symposium on the Accelerated Cooling of Steel; 1985; Pittsburgh, EUA. Englewood: AIME; 1985. p. 18I-194.

4 Ludwig B. Systems for the accelerated cooling of plates. Metallurgical Plant and Technology. 1988;16:10-17.

5 Ouchi C. Development of steel plates by intensive use of TMCP and direct quenching process. ISI] International. 200I; I 4(6):542-553. http://dx.doi.org/I0.2355/isijinternational.4I.542

6 Wang SC, Chiu FJ. Establishment of TMCP process in the plate mill of CSC. In: Proceedings of the Accelerated Cooling/Direct Quenching Steels; 1997; Indianapolis, EUA. Michigan: ASM International; 1997. p. 77-86.

7 Evans JF, Clark MT. Plate cooling: technologies and market requirements. AISE Steel Technology. 2002;79(6):49-53.

8 Schmidt R, Dehmel R, Horn G. Latest technologies in plate cooling and their benefit in plate production. Revue de Métallurgie. 2008; I05(5):280-285. http://dx.doi.org/I0.105I/metal:200804 I

9 Bodnar RL, Shen Y, Lin M, Elwood DW, Feher FC, Roe GJ. Accelerated cooling on Burns Harbor's I60" plate mill. In: Proceedings of the Accelerated Cooling/Direct Quenching Steels; 1997; Indianapolis, EUA. Michigan: ASM International; 1997. p. 3-13.

10 Santos AA, Schiavo CP, Giacomin CN. Simulação computacional do processo de reaquecimento de placas em fornos de viga móvel. Tecnologia em Metalurgia e Materiais. 2008;5(I):35-39. http://dx.doi.org// 0.4322/tmm.0050I 007

I I Giacomin CN, Santos AA, Souza AL. Análise dos processos de laminação a quente na Usiminas via simuladores computacionais. Tecnologia em Metalurgia e Materiais. 2009;6(I):3I-35. http://dx.doi.org/I0.4322/tmm.0060I 006

12 Santos AA, Giacomin CN. Mathematical simulation of plate rolling at Usiminas: a tool for process enhancement. In: Anales de la $18^{a}$ Conferencia de Laminación; 2010; Rosario, Argentina. Buenos Aires: Instituto Argentino de Siderurgia; 2010.

I3 Bingxing W, Zhaodong W, Guodong W. Research of the new generation TMCP technologies for high performance steel plates. In: Proceedings of the 6th European Rolling Conference; 20I3; Venice, Itália. Milano: Associazione Italiana de Metallurgia; 2013. 
I4 Chalmers M, Robinson I, Samanta S, Hulley M. Control of plate themomechanical properties using MULPIC plate cooling technology. In: Proceedings of the 6th European Rolling Cionference; 2013; Venice, Itália. Milano: Associazione Italiana de Metallurgia; 2013.

15 The British Iron and Steel Association. Physical constants of some commercial steels at elevated temperatures. London: Butterworths; 1953.

16 Carnahan B, Luther HA, Wilkes JO. Applied numerical methods. New York: John Wiley \& Sons; 1969.

17 Patankar SV. Numerical heat transfer and fluid flow. New York: McGraw-Hill; 1980.

18 Panjkivic V, Gloss R. Fast dynamic heat and mass balance model of walking beam reheat furnace with two-dimensional slab temperature profile. Ironmaking and Steelmaking. 2012;39(3): 190-209. http://dx.doi.org/I0.I I 79/|74328I2IIY.000000008I

19 Incropera FP, De Witt DP. Fundamentos de transferência de calor e massa. Rio de Janeiro: LTC; 2003. http://dx.doi. org/I0.1I79/174328/21IY.000000008।

20 Goldbarg MC, Luna HPL. Otimização combinatória e programação linear: modelos e algoritmos. Rio de Janeiro: Campus; 2005.

Recebido em: 20 Mar. 2014

Aceito em: 10 Jun. 2014 